\title{
FLIP: Benchmark tasks in fitness landscape inference for proteins
}

\author{
Christian Dallago* \\ Technical University of Munich \\ christian.dallago@tum.de
}

\author{
Jody Mou* \\ Microsoft Research New England \\ jodymou@mit.edu
}

\author{
Kadina E. Johnston \\ BBE, Caltech \\ kjohnston@caltech.edu
}

bwittman@caltech.edu

\author{
Nicholas Bhattacharya \\ UC Berkeley \\ nick_bhat@berkeley.edu
}

\author{
Samuel Goldman \\ CSB, MIT \\ samlg@mit.edu
}

\author{
Ali Madani \\ Saleforce Research \\ amadani@salesforce.com
}

\author{
Kevin K. Yang \\ Microsoft Research New England \\ yang.kevin@microsoft.com
}

\begin{abstract}
Machine learning could enable an unprecedented level of control in protein engineering for therapeutic and industrial applications. Critical to its use in designing proteins with desired properties, machine learning models must capture the protein sequence-function relationship, often termed fitness landscape. Existing benchmarks like CASP or CAFA assess structure and function predictions of proteins, respectively, yet they do not target metrics relevant for protein engineering. In this work, we introduce Fitness Landscape Inference for Proteins (FLIP), a benchmark for function prediction to encourage rapid scoring of representation learning for protein engineering. Our curated tasks, baselines, and metrics probe model generalization in settings relevant for protein engineering, e.g. low-resource and extrapolative. Currently, FLIP encompasses experimental data across adeno-associated virus stability for gene therapy, protein domain B1 stability and immunoglobulin binding, and thermostability from multiple protein families. In order to enable ease of use and future expansion to new tasks, all data are presented in a standard format. FLIP scripts and data are freely accessible at https://benchmark.protein.properties.
\end{abstract}

\section{Introduction}

Proteins are life's workhorses, efficiently and precisely performing complex tasks under a wide variety of conditions. This combination of versatility and selectivity makes them not only critical to life, but also to a myriad of human-designed applications. Engineered proteins play increasingly essential roles in industries and applications spanning pharmaceuticals, agriculture, specialty chemicals, and fuel [1-5]. The ability of a protein to perform a desired function is determined by its amino acid sequence, often mediated through folding to a three-dimensional structure [6]. Unfortunately, current biophysical and structural prediction methods cannot reliably map a sequence to its ability to perform a desired function, termed protein fitness, with sufficient precision to distinguish between closely-related protein sequences performing complex functions such as catalysis. Therefore, protein engineering has relied heavily on directed evolution (DE) methods, which stochastically modify ("mutate") a starting sequence to create a library of sequence variants, measure all variants to find those with improved fitness, and then iterate until the protein is sufficiently optimized [7]. Directed evolution is energy-, time-, and material-intensive, in part because it discards information from

* Equal contribution 
unimproved sequences. Machine-learning methods that predict fitness from sequence can leverage both positive and negative data to intelligently select variants for screening, reaching higher fitness levels with fewer measurements than traditional directed evolution, and without necessarily requiring detailed understanding of structure or mechanism [8, 7, 9-11].

Directed evolution campaigns are often limited by the cost of collecting sequence-fitness data. Therefore, machine learning approaches for sequence-fitness prediction are most useful in protein engineering when they can learn from low- $\mathrm{N}$ (few sample) labeled datasets or when they can generalize to types of variation that are unobserved in the training set. Rapid advances in genomic sequencing technology have led to an explosion of putative protein sequences [12, 13] deposited in databases like UniProt [14]. Recent efforts in sequence-function prediction [15, 16] have sought to leverage the information in these unlabeled sequences through pretraining and fine-tuning, and have successfully engineered proteins with brighter fluorescence and high catalytic efficiency [17]. Unsupervised models were also applied to- or built on evolutionary sequence inputs to model the effects of mutations [18-21].

In this work, we present a suite of benchmarking tasks for protein sequence-fitness prediction with the dual aims of enabling protein engineers to compare and choose machine learning methods representing protein sequences and accelerating research on machine learning for protein fitness prediction. Our tasks are curated to be diverse in the functions measured and in the types of underlying sequence variation. For each landscape, we provide one or more train/test splits that evaluate biologically-relevant generalization and mimic challenges often seen in protein engineering. Figure 1 and Table 2 summarize the landscape tasks and splits. We also compute the performance of baseline models against which future models can be compared, and which highlight that our tasks can distinguish between "better" and "worse" pretraining regimes. Landscapes and baselines are available at https://benchmark.protein.properties, while a glossary technical terms is provided in the supplement.

\section{Related Work}

Well-designed and easily accessible benchmarks have encouraged and measured progress in machine learning on proteins, especially protein structure prediction. The Critical Assessment of Protein Structure Prediction (CASP) [22], and retrospective protein training datasets from previous CASP competitions [23] have lowered the barrier to entry for new research teams and provided a clear account of progress over the last three decades [24]. DeepMind's recent landmark results with their AlphaFold2 predictor in CASP 14 [25] built on these community-driven efforts.

Table 1: Performance (Spearman's correlation) on TAPE engineering tasks. Performances reported in referenced literature.

\begin{tabular}{llrr}
\hline & Pretraining & Fluorescence & Stability \\
\hline ESM [26] & masked language model & 0.68 & 0.71 \\
\hline TAPE transformer [27] & masked language model & 0.68 & 0.73 \\
TAPE LSTM [27] & bidirectional language model & 0.67 & 0.69 \\
TAPE ResNet [27] & masked language model & 0.21 & 0.73 \\
UniRep [28] & language model + structure & 0.67 & 0.73 \\
\hline CPCProt [29] & contrastive & 0.68 & 0.65 \\
CPCProt-LSTM [29] & contrastive & 0.68 & 0.68 \\
\hline Linear regression [30] & none & 0.68 & 0.48 \\
CNN [30] & none & 0.67 & 0.51 \\
Mutation count [31] & none & 0.45 & NA \\
BLOSUM62 score [31] & none & 0.50 & NA \\
\hline
\end{tabular}

Inspired by the effectiveness of CASP, there have been attempts at benchmarks for function prediction and protein pretraining. The Critical Assessment of Function Annotation (CAFA) [32, 33] focuses on assigning Gene Ontology (GO) classes (categorical definitions of protein functions) to proteins. While an important benchmark, CAFA does not directly require models to build on sequence inputs, instead they could leverage graph inputs from protein-protein interaction networks, and the prediction targets 
do not account for fitness variations between very similar sequences that are important for protein engineering. Tasks Assessing Protein Embeddings (TAPE) [27] aims to evaluate the effectiveness of different pretraining regimes and models to predict protein properties. Of the five tasks in TAPE, three (remote homology, secondary structure, and contacts) focus on structure prediction, while only two (fluorescence and stability) target fitness prediction. These two tasks show little discriminative power between different models [30], as shown in Table 1. In addition, the use of structure as an evaluation limits the creation of jointly trained structure- and sequence- based embeddings that may be most useful in protein engineering tasks [34]. Envision [35] collates several dozen single amino-acid variation (SAV) datasets, but does not include other types of sequence variation of interest to protein engineers. DeepSequence [19] collects 42 deep mutational scan (DMS) datasets for evaluation purposes. These capture single and multiple co-occurring residue substitutions, but do not capture variation at the proteome scale, or mutational paths from large insertions and deletions. Furthermore, while DMS landscapes may characterize the effect of co-occurring substitutions, not every sample with co-occurring residue substitutions may express these at sites relevant for a measured function, and in turn, evaluations on all possible co-occurring substitutions may not always be expressive (e.g., if the measured function is binding and a sample has two substitutions, one at a residue at the interface and one elsewhere, the effect may still be high simply because an interface residue is involved). Finally, the data from these studies does not come with standard column headers or train/test splits, hindering use in automated evaluation pipelines.

The limitations of the existing benchmarks have led pretraining methods to be primarily evaluated by their ability to predict structural information [36, 37]. While the ability to impart structural knowledge through sequence-only pretraining is impressive, it is not the most important criterion for protein engineers. Efforts to systematically compare new methods on fitness prediction have required researchers to both gather their own collection of datasets and compute their own baseline comparisons [16, 38-40].

\section{Landscapes and Splits}

We design FLIP to answer two fundamental questions about machine learning model learning protein sequences:

1. Can a model capture complex fitness landscapes beyond mutations to a parent sequence?

2. Can a model perform well across a range of proteins where fitness is measured for very different functions?

Existing work such as DeepSequence [19] and Envision [35] succeed at the second criterion but not the first. TAPE [27], on the other hand, evaluates the first criterion with its fluorescence task but not the second. We prioritized complex landscapes (with insertions and deletions) rather than single amino acid variants (e.g. deep mutational scans), to practically cover a larger sequence space, as well as potentially more functional diversity finalized to ensure model generalization for broad applicability.

To test the aforementioned questions, we collect three published landscapes and create 15 corresponding dataset splits as desribed in the following and summarized in Table 2 . We choose landscapes and splits that cover a broad range of protein families, sequence variation, and fitness landscapes with rigorous measurements. Each landscape is transformed into one or more splits to test different model generalization abilities, as shown in Figure $1 ;$; many of the splits were also made to reflect standard laboratory data-collection practices, thus testing the appropriateness of models to real-world applications.

Simple random splits are notoriously misleading in classical protein sequence-to-function prediction as protein sequences are not sampled I.I.D., but with correlations induced by evolutionary history. This means that random splits reflect a notion of generalization not of interest to most biologists [46]. While there are standard heuristics for approximating the correlation structure due to evolution (such as sequence-identity deduplication/redundancy reduction), in the protein engineering setting there are not similarly standardized approaches. As such, we resorted to landscape-specific approaches informed by the conditions of each experiment, as detailed in Figure 1 . 


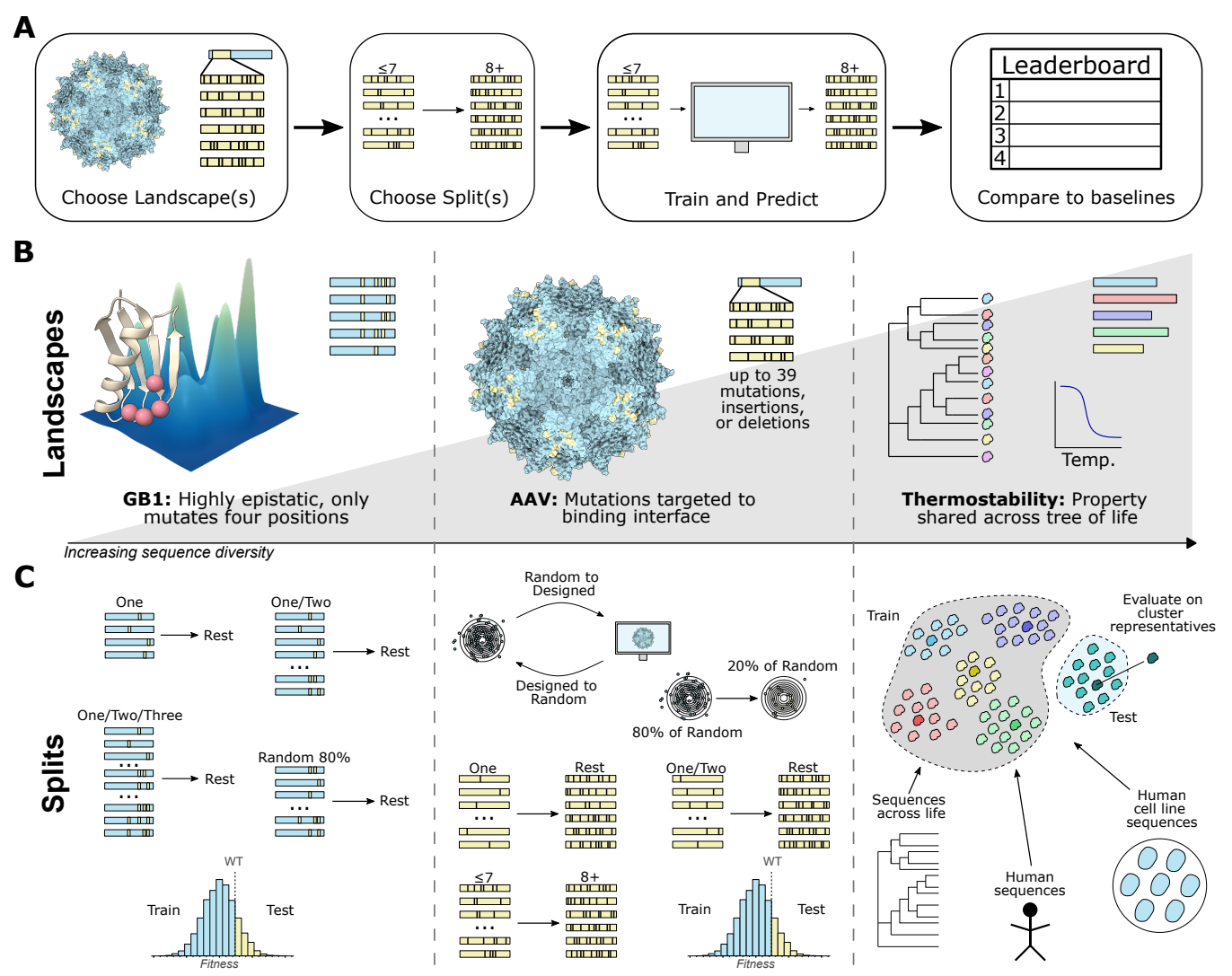

Figure 1: Summary of the workflow, landscapes, and splits. (A) General FLIP workflow: choose landscapes and splits that match user needs, train models and make predictions on the test set, and then compare to baseline models. (B) We choose landscapes that cover different types of sequence diversity. The GB1 landscape focuses on simultaneous mutation of four epistatic sites with nearly complete coverage [41] (PDB ID: 2GI9 [42]). The AAV capsid protein landscape sparsely samples sequences with up to 28 mutations, including insertions and deletions, to the the binding interface [43] (PDB ID: 6IH9 [44]). The thermostability landscape [45] measures a property shared by proteins from multiple functional groups across different domains of life. (C). We also provide up to seven suggested data splits for each landscape, which are described in Section 3 .

Table 2: Landscapes and split statistics. The sampled splits $(*)$ are mainly used for discourse in this manuscript, as such splits are rarely observed in practice when working with biological data.

\begin{tabular}{llrrr}
\hline Landscape & Split & Total samples & Train samples & Test samples \\
\hline AAV & Mut-Des & 284,009 & 82,583 & 201,426 \\
& Des-Mut & $284^{\prime} 009$ & 201,426 & 82,583 \\
& 1-vs-rest & 82,583 & 1,170 & 81,413 \\
& 2-vs-rest & 82,583 & 31,807 & 50,776 \\
& 7-vs-rest & 82,583 & 70,002 & 12,581 \\
& low-vs-high & 82,583 & 47,546 & 35,037 \\
\multirow{5}{*}{ Thermostability } & Sampled* & 82,583 & 66,066 & 16,517 \\
& Mixed & 27,951 & 24,817 & 3,134 \\
& Human & 10,093 & 8,148 & 1,945 \\
GB1 & Human-cell & 7,156 & 5,792 & 1,366 \\
& 1-vs-rest & 8,733 & 29 & 8,704 \\
& 2-vs-rest & 8,733 & 427 & 8,306 \\
& 3-vs-rest & 8,733 & 2,968 & 5,765 \\
& low-vs-high & 8,733 & 5,089 & 3,644 \\
& Sampled* & 8,733 & 6,961 & 1,772 \\
\hline
\end{tabular}


The vast majority of representation learning on protein sequences models entire sequences [26, 37, [15, 34]. As such, we use entire protein sequences as inputs, even for landscapes derived from studies examining mutations at a small subset of positions. While we include a naïve validation set for each split for comparison purposes, we encourage users to engineer their own validation splits from the training data. All tasks and splits are provided in a consistent, easy-to-use CSV format and are available at https://benchmark.protein.properties. Original datasets were either supplemented to published research (Wu et al.) under CC BY 4.0, or were obtained with written permission from the authors (Jarzab et al., Bryant et al.). Data derivatives proposed as tasks are licensed under AFL-3.

\subsection{GB1}

Motivation. One challenge confronting protein engineering is the ability to predict the effects of interactions between mutations, termed epistasis. These interactions result in non-additive effects on protein fitness and have been shown to constrain the paths available to evolution, especially evolution via a greedy walk. Furthermore, as more mutations are made simultaneously, these interactions become more complex and more difficult to predict. Therefore, we wish to assess model predictions on an exhaustive, combinatorial, and highly epistatic mutational landscape, focusing on learning from variants with fewer mutations to predict the activity of variants with more mutations.

Landscape. We use the GB1 landscape [41], which has become a gold standard for investigating epistatic interactions [10]. GB1 is the binding domain of protein G, an immunoglobulin binding protein found in Streptococcal bacteria [47, 48]. In their original study, Wu et al. measured the fitness of 149, 361 of 160,000 possible combinations of mutations at 4 positions.

Splits. Over $96 \%$ of the amino acid mutations in this set yield non- or poorly-binding sequences $-143,539$ out of 149,361 sequences have fitness value below 0.5 , where wild-type fitness is 1 and a fitness of 0 is non-binding. Thus, models trained on the full experimental data can achieve high performance by predicting low fitness regardless of inputs. To ensure that models learn nontrivial signal, we downsample non-functional sequences prior to creating the training sets. Specifically, we include all 5822 sequences with fitness above 0.5 and 2911 randomly-sampled sequences with fitness less than or equal to 0.5 . From this set, we curate five dataset splits to test generalization from few-mutation sequences to many-mutation sequences, from low fitness to high, and one extra randomly sampled split for discussion purposes:

- Train on single mutants (1-vs-rest): Wild type and single mutants are assigned to train, while the rest are assigned to test. This split is one of the most commonly observed in an applications setting, where a researcher has gathered data for many single mutations of interest and wishes to predict the best combinations of mutations.

- Train on single and double mutants (2-vs-rest): Wild type, single and double mutants are assigned to train, while the rest are assigned to test. This is also a commonly observed split in an applications setting, albeit, at a lesser frequency than 1-vs-rest.

- Train on single, double and triple mutants (3-vs-rest): Wild type, single, double and triple mutants are assigned to train, while the rest are assigned to test.

- Train on low fitness, test on high (low-vs-high): Sequences with fitness value equal or below wild type are used to train, while sequences with fitness value above wild type are used to test.

- Sampled: Sequences are randomly partitioned in $80 \%$ train and $20 \%$ test. This split serves mostly for discussion purposes in this manuscript.

\subsection{AAV}

Motivation. Mutations for engineering are often focused in a specific region of a protein. For example, this is done if a protein-protein interface is known to be at a subset of positions. Successfully predicting fitness for a long sequence being mutated at a subset of positions is a task of wide applicability. 
Landscape. Adeno-associated virus (AAV) capsid proteins are responsible for helping the virus integrate a DNA payload into a target cell [49], and there is great interest in engineering versions of these proteins for gene therapy [43, 50, 51]. Bryant et al. prepared a rich mutational screening landscape of different VP-1 AAV proteins (UniProt [14] Accession: P03135), and this data has been successfully used as a basis for machine learning-guided design [52, 53]. In their study, Bryant et al. mutagenize a 28 -amino acid window from position 561 to 588 of VP-1 and measure the fitness of resulting variants with between 1 and 39 mutations, which we refer to as the sampled pool. In addition they measured the fitness of sequences chosen or designed using various machine-learning models. We refer to these as the designed pool.

Splits. We derive seven splits from this landscape that probe model generalization:

- Sampled-designed (Mut-Des): All sampled sequences are assigned to train; all designed sequences are assigned to test.

- Designed-sampled (Des-Mut): All designed sequences are assigned to train; all sampled sequences are assigned to test.

- Train on single mutants (1-vs-rest): Wild type and single mutants in the sampled pool are assigned to train, while the rest are assigned to test. As with the GB1 1-vs-rest split, this reflects a common dataset split observed in protein engineering applications.

- Train on single and double mutants (2-vs-rest): Wild type, single and double mutants in the sampled pool are assigned to train, while the rest are assigned to test. Again, as with the GB1 2-vs-rest split, this reflects a common dataset split observed in protein engineering applications.

- Train on mutants with up to seven changes (7-vs-rest): Mutants with up to and including seven changes in the sampled pool are assigned to train, while the rest are assigned to test.

- Train on low fitness, test on high (low-vs-high): For sequences in the in the sampled pool, sequences with fitness value equal or below wild type are used to train, while sequences with fitness value above wild type are used to test.

- Sampled: Sequences in the sampled pool are randomly partitioned in $80 \%$ train and $20 \%$ test. This split serves mostly for discussion purposes in this manuscript.

\subsection{Thermostability}

Motivation. Thermostability is very often a desirable trait that complements more applicationspecific functions. For example, thermostable enzymes not only allow operation at higher reaction temperatures with faster reaction rates, but are also better starting points for directed evolution campaigns [54, 55]. This explains why thermostability has been a consistent target for multi-objective optimization in protein engineering [56-58]. Thermostability can be challenging to predict, because it is not necessarily a smooth function landscape; in certain protein families, a single amino acid substitution can confer or destroy thermostability [59].

Landscape. We curate an extensive screening landscape from the Meltome Atlas [45], which used a mass spectrometry-based assay to measure protein melting curves across 13 species and 48,000 proteins. Unlike the other landscapes, which measure the effects of sequence variation from a single starting point on a function specific to that protein, this landscape includes both global and local variation.

Splits. We derive three splits from this landscape, considering biological realities and common dataset regularizations for cross-spices and sequence-diverse sets:

- Mixed: We cluster all available sequences and select cluster representatives using MMseqs2 [12] at a threshold of $20 \%$ sequence identity to create one split. In this split, all sequences in $80 \%$ of clusters are assigned to train, while only cluster representatives from the remaining $20 \%$ of clusters are assigned to test.

- Human: We cluster sequences in human and select cluster representatives using MMseqs2 [12] at a threshold of $20 \%$ sequence identity to create one split. In this split, all 
sequences in $80 \%$ of clusters are assigned to train, while only cluster representatives from the remaining $20 \%$ of clusters are assigned to test.

- Human-cell: We cluster sequences of one cell line for human and select cluster representatives using MMseqs2 [12] at a threshold of $20 \%$ sequence identity to create one split. In this split, all sequences in $80 \%$ of clusters are assigned to train, while only cluster representatives from the remaining $20 \%$ of clusters are assigned to test.

\section{Baseline algorithms}

We evaluate three major groups of baselines (Table $\sqrt{3}$ ) - parameter-free, supervised, and pretrained. These three classes correspond to common approaches from different communities. In particular, we seek to clarify the value of transfer learning for protein engineering by benchmarking pretrained models against purely supervised methods systematically. We also hope to simplify algorithm selection for practitioners by providing a single place to compare many commonly used methods. Note that we do not use Potts models [60], popular in protein structure prediction [61], because of the need to build high-quality multiple sequence alignments, which would be impractical for the thermostability dataset. Furthermore, Potts models use artificial constructs when dealing with datasets with large insertions and deletions (e.g., modeling sequence deletions through special characters), as is the case for the AAV landscape. However, in the presence of well curated MSAs, these approaches can be successful in modeling the effect of residue substitutions [62].

Table 3: Baseline methods

\begin{tabular}{ll}
\hline Method & Description \\
\hline Levenshtein & Levenshtein distance to wild-type. \\
BLOSUM62 & BLOSUM62-score relative to wild-type. \\
Ridge regression & Ridge regression model on one-hot encoding. \\
Convolutional network & Simple convolutional network on one-hot encoding. \\
ESM-untrained & 750M parameter transformer with randomly-initialized weights \\
ESM-1b [26] & 750M parameter transformer pretrained on UniRef50. \\
ESM-1v [16] & 750M parameter transformer pretrained on UniRef90. Only one \\
& element of ensemble used due to compute constraints. \\
\hline
\end{tabular}

For baselines using protein language models, which compute an embedding for every amino acid, we pool embeddings in three ways:

- Per amino acid (per AA): A supervised model is tasked to learn how to pool over the sequence using a $1 \mathrm{D}$ attention layer to return a regression prediction.

- Mean: Sequence embeddings are mean pooled per amino acid over the length of the protein sequence to obtain a fixed-size input for each sequence.

- Mean over subset (mut mean): Sequence embeddings are mean pooled per amino acid for the residues in the mutated region of interest to obtain a fixed-size, region specific input from the sequence.

To train the models, $10 \%$ of each training set is sampled at random as a validation set. For Ridge, we use the scikit-learn implementation of ridge regression with default parameters. The CNN consists of a convolution with kernel width 5 and 1024 channels, a ReLU non-linearity, a linear mapping to 2048 dimensions, max pool over the sequence, and a linear mapping to 1 dimension. CNNs are optimized using Adam [63] with a batch size of 256 (GB1, AAV) or 32 (thermostability) and a learning rate of 0.001 for the convolution weights, 0.00005 for the first linear mapping, and 0.000005 for the second linear mapping. Both linear mappings have a weight decay of 0.05. For ESM models, by far the most computationally expensive baselines, we train with a batch size of 256 , a learning rate of 0.001 , and the Adam optimizer. CNNs and the ESM models are trained with early stopping with a patience of 20 epochs. Models are trained on a NVidia Quadro RTXA6000 GPU. Code, data, and instructions needed to reproduce results can be found at https://benchmark.protein.properties. 


\section{Results}

Overall, we observe that for landscapes around a wild type (Tables $4 \&[5$ ), pretraining offered by ESM-1b [26] or ESM-1v [16] does not help much when sufficient training data is available (see Table 2 for statistics), at least in the setting explored here: using these protein language models to collect frozen embeddings as inputs to subsequent prediction models. Conversely, for the split involving diverse sequences (Table 6), pretraining yields a large boost over pure supervision. The best method of pooling residue-embeddings for whole sequences varies depending on task (Table 4, 5, \& 6). Most remarkably, training simple models (CNN, ridge regression) is competitive over a wide range of regimes. We exclude results for per-AA ESM models for the AAV Des-Mut task (Table 5), as we estimated that it would require a month of compute using for Nvidia A6000 GPUs, which appeared unjustified for a baseline metric computation. Hyperparameter search results are reported in the supplement, as are evaluations using different metrics.

Table 4: GB1 baselines (metric: Spearman correlation)

\begin{tabular}{lcccc}
\hline Model & 1-vs-rest & 2-vs-rest & 3-vs-rest & low-vs-high \\
\hline ESM-1b (per AA) & 0.28 & 0.55 & 0.79 & 0.59 \\
ESM-1b (mean) & 0.32 & 0.36 & 0.54 & 0.13 \\
ESM-1b (mut mean) & -0.08 & 0.19 & 0.49 & 0.45 \\
ESM-1v (per AA) & 0.28 & 0.28 & 0.82 & 0.51 \\
ESM-1v (mean) & 0.32 & 0.32 & 0.77 & 0.10 \\
ESM-1v (mut mean) & 0.19 & 0.19 & 0.80 & 0.49 \\
\hline ESM-untrained (per AA) & 0.06 & 0.06 & 0.48 & 0.23 \\
ESM-untrained (mean) & 0.05 & 0.05 & 0.46 & 0.10 \\
ESM-untrained (mut mean) & 0.21 & 0.21 & 0.57 & 0.13 \\
Ridge & 0.28 & 0.59 & 0.76 & 0.34 \\
CNN & 0.17 & 0.32 & 0.83 & 0.51 \\
\hline Levenshtein & 0.17 & 0.16 & -0.04 & -0.10 \\
BLOSUM62 & 0.15 & 0.14 & 0.01 & -0.13 \\
\hline
\end{tabular}

GB1. Table 4 summarizes baseline results for the biologically motivated GB1 splits. When models are trained only on single mutants, all variations on ESM-1b [26] and ESM-1v [16] outperform supervised models. This regime has little training data (29 samples, Table 2), giving the most opportunity for pretraining to compensate. The difference between pretrained and supervised models largely disappears once models are trained on both single and double mutants (2-vs-rest, Table 4). The various pooling choices for embeddings perform inconsistently across datasets and splits; for example, mut-mean does best on 1-vs-rest but worst on 3-vs-rest. The low-vs-high split suggests. The sampled split reported separately in Table 7 confirms: random sampling sequences in biology is bound to overestimate results.

AAV. Table 5 summarizes baseline results for the biologically motivated AAV splits. Across all splits, purely supervised models are competitive with pretrained models. This suggests that the large sizes of training sets are past the threshold where pretraining improves performance. The particular choice of pooling that performs best is inconsistent across splits. The BLOSUM62 baseline could not be applied as the mutations in this set include insertions and deletions. In this case too, the sampled split reported separately in Table 7 strongly suggest that random sampling sequences in biology may lead to overestimated results.

Thermostability. Table 6 summarizes baseline results for thermostability. Pretrained models consistently outperform supervised models on this task, suggesting that this landscape is not yet past the threshhold where pretraining improves performance. Interestingly, the supervised baselines based on untrained ESM embeddings do better than either ridge or CNN. Mean over subset (mut mean) and BLOSUM62 are not applicable for the Meltome landscape as the sequences are not evolutionarily related. 
Table 5: AAV baselines (metric: Spearman correlation)

\begin{tabular}{lcccccc}
\hline \multicolumn{1}{c}{ Model } & Mut-Des & Des-Mut & 1-vs-rest & 2-vs-rest & 7-vs-rest & low-vs-high \\
\hline ESM-1b (per AA) & 0.76 & - & 0.03 & 0.65 & 0.65 & 0.39 \\
ESM-1b (mean) & 0.63 & 0.59 & 0.04 & 0.26 & 0.46 & 0.18 \\
ESM-1b (mut mean) & 0.70 & 0.70 & 0.31 & 0.65 & 0.61 & 0.33 \\
ESM-1v (per AA) & 0.79 & - & 0.10 & 0.70 & 0.70 & 0.34 \\
ESM-1v (mean) & 0.55 & 0.44 & 0.18 & 0.16 & 0.45 & 0.20 \\
ESM-1v (mut mean) & 0.70 & 0.71 & 0.44 & 0.64 & 0.64 & 0.31 \\
\hline ESM-untrained (per AA) & 0.56 & - & 0.18 & 0.22 & 0.42 & 0.08 \\
ESM-untrained (mean) & 0.27 & 0.34 & 0.01 & 0.14 & 0.22 & 0.22 \\
ESM-untrained (mut mean) & 0.62 & 0.64 & 0.26 & 0.16 & 0.56 & 0.24 \\
Ridge & 0.64 & 0.53 & 0.22 & 0.03 & 0.65 & 0.12 \\
CNN & 0.71 & 0.75 & 0.48 & 0.74 & 0.74 & 0.34 \\
\hline Levenshtein & 0.60 & -0.07 & -0.11 & 0.57 & 0.53 & 0.25 \\
BLOSUM62 & NA & NA & NA & NA & NA & NA \\
\hline
\end{tabular}

Table 6: Thermostability baselines (metric: Spearman correlation)

\begin{tabular}{lccc}
\hline Model & Mixed & Human & Human-Cell \\
\hline ESM-1b (per AA) & 0.68 & 0.71 & 0.76 \\
ESM-1b (mean) & 0.68 & 0.70 & 0.75 \\
ESM-1b (mut mean) & NA & NA & NA \\
ESM-1v (per AA) & 0.65 & 0.77 & 0.78 \\
ESM-1v (mean) & 0.67 & 0.75 & 0.74 \\
ESM-1v (mut mean) & NA & NA & NA \\
\hline ESM-untrained (per AA) & 0.44 & 0.44 & 0.46 \\
ESM-untrained (mean) & 0.36 & 0.48 & 0.49 \\
ESM-untrained (mut mean) & NA & NA & NA \\
Ridge & 0.17 & 0.15 & 0.24 \\
CNN & 0.34 & 0.50 & 0.49 \\
\hline Levenshtein & NA & NA & NA \\
BLOSUM62 & NA & NA & NA \\
\hline
\end{tabular}

\section{Discussion}

The prediction tasks in FLIP probe complex fitness landscapes across different protein functions. We curate three landscapes published in existing literature and formulate 15 corresponding splits of the data to mimic protein engineering tasks. The main criteria to include a landscape was whether it could be used to assess interesting types of generalization, and if it was amenable to interpretable assessment metrics. As no standard approach exists to partition landscapes arising from mutagenesis of a parent sequence, we propose ideas that may be applied to future landscapes. In particular, we explore the concept of training on sequences only a few mutations from a parent while predicting on data many mutations from a parent in a step-by-step fashion.

The need for more challenging splits is illustrated in Table 7, which shows results for the sampled splits, based on simple random sampling. Almost all models do drastically better for the sampled splits, and differences between models are exaggerated. This indicates the importance of biologicallymotivated generalization in task design.

In general, results on baselines highlight that while pretraining approaches perform well on tasks with diverse sequences (Thermostability, Table 6), they do not outperform simpler models on mutational landscapes (GB1, Table $4 \&$, AAV, Table 5). In addition, large pretrained models require amounts of compute (up to 50 days on an NVidia A6000 GPU) to train on some tasks, which is out of the reach of most academic research groups. It is important to note that while we performed a modest hyperparameter search, more extensive sweeps combined with training data regularization 
Table 7: Optimistic results for random splits (Sampled) on the AAV and GB1 sets (metric: Spearman correlation)

\begin{tabular}{lcc}
\hline Landscape & AAV & GB1 \\
\hline ESM-1b (per AA) & 0.90 & 0.92 \\
ESM-1v (per AA) & 0.92 & 0.92 \\
\hline ESM-untrained (per AA) & 0.78 & 0.79 \\
Ridge & 0.83 & 0.82 \\
CNN & 0.92 & 0.91 \\
\hline
\end{tabular}

like different validation splits, may yield better absolute and relative performance. The landscapes and derived prediction splits offered in FLIP highlight directions for future work, such as better pretraining or embedding methods for protein mutational landscapes.

\section{Conclusion}

The proliferation of protein sequence data, along with advanced experimental techniques for functional measurement of proteins, presents a ripe environment for machine learning-enabled solutions in protein engineering. With the introduction of FLIP, we focus on sequence-fitness prediction and aim to encourage rigorous evaluation of model generalization in multiple tasks and settings relevant to protein engineering. We hope to seed advances in this emerging interdisciplinary field with downstream applications for solutions in human health and the environment. FLIP data and scripts are available under free licenses at https://benchmark.protein.properties.

\section{Acknowledgments and Disclosure of Funding}

The authors thank Jeffrey Spencer, Sam Sinai, Sam Bowman, Roshan Rao and Debora Marks for ideas and discussions that helped us improve our work. The authors would also like to thank Helix and Murphy for careful attention to the manuscript. C.D. acknowledges support from the Bundesministerium für Bildung und Forschung (BMBF) - Project numbers: 01IS17049 and 031L0168. K.E.J. and B.J.W. acknowledge the NSF Division of Chemical, Bioengineering, Environmental and Transport Systems (1937902). N.B. was supported in part by NIH grant R35-GM134922 and by the Exascale Computing Project (17-SC-20-SC), a collaborative effort of the U.S. Department of Energy Office of Science and the National Nuclear Security Administration. S.G. thanks the MIT Machine Learning for Pharmaceutical Discovery and Synthesis Consortium for supporting this work. K.K.Y. was previously employed by Generate Biomedicines.

\section{References}

[1] Stephan Luetz, Lori Giver, and James Lalonde. Engineered enzymes for chemical production. Biotechnology and Bioengineering, 101(4):647-653, August 2008. doi: 10.1002/bit.22077. URL https://doi.org/10.1002/bit.22077. 1

[2] Fei Wen, Nikhil U Nair, and Huimin Zhao. Protein engineering in designing tailored enzymes and microorganisms for biofuels production. Current Opinion in Biotechnology, 20(4):412419, August 2009. doi: 10.1016/j.copbio.2009.07.001. URL https://doi.org/10.1016/j. copbio.2009.07.001.

[3] Swati Kapoor, Aasima Rafiq, and Savita Sharma. Protein engineering and its applications in food industry. Critical Reviews in Food Science and Nutrition, 57(11):2321-2329, June 2015. doi: 10.1080/10408398.2014.1000481. URL https://doi.org/10.1080/10408398.2014. 1000481 .

[4] Mark A. Huffman, Anna Fryszkowska, Oscar Alvizo, Margie Borra-Garske, Kevin R. Campos, Keith A. Canada, Paul N. Devine, Da Duan, Jacob H. Forstater, Shane T. Grosser, Holst M. Halsey, Gregory J. Hughes, Junyong Jo, Leo A. Joyce, Joshua N. Kolev, Jack Liang, Kevin M. Maloney, Benjamin F. Mann, Nicholas M. Marshall, Mark McLaughlin, Jeffrey C. Moore, 
Grant S. Murphy, Christopher C. Nawrat, Jovana Nazor, Scott Novick, Niki R. Patel, Agustina Rodriguez-Granillo, Sandra A. Robaire, Edward C. Sherer, Matthew D. Truppo, Aaron M. Whittaker, Deeptak Verma, Li Xiao, Yingju Xu, and Hao Yang. Design of an in vitro biocatalytic cascade for the manufacture of islatravir. Science, 366(6470):1255-1259, 2019. ISSN 00368075. doi: 10.1126/science.aay8484. URL https://science.sciencemag.org/content/ $366 / 6470 / 1255$.

[5] Carlos Eduardo Sequeiros-Borja, Bartłomiej Surpeta, and Jan Brezovsky. Recent advances in user-friendly computational tools to engineer protein function. Briefings in Bioinformatics, 22 (3), July 2020. doi: 10.1093/bib/bbaa150. URL https://doi.org/10.1093/bib/bbaa150. [1]

[6] Predrag Radivojac, Wyatt T Clark, Tal Ronnen Oron, Alexandra M Schnoes, Tobias Wittkop, Artem Sokolov, Kiley Graim, Christopher Funk, Karin Verspoor, Asa Ben-Hur, Gaurav Pandey, Jeffrey M Yunes, Ameet S Talwalkar, Susanna Repo, Michael L Souza, Damiano Piovesan, Rita Casadio, Zheng Wang, Jianlin Cheng, Hai Fang, Julian Gough, Patrik Koskinen, Petri Törönen, Jussi Nokso-Koivisto, Liisa Holm, Domenico Cozzetto, Daniel W A Buchan, Kevin Bryson, David T Jones, Bhakti Limaye, Harshal Inamdar, Avik Datta, Sunitha K Manjari, Rajendra Joshi, Meghana Chitale, Daisuke Kihara, Andreas M Lisewski, Serkan Erdin, Eric Venner, Olivier Lichtarge, Robert Rentzsch, Haixuan Yang, Alfonso E Romero, Prajwal Bhat, Alberto Paccanaro, Tobias Hamp, Rebecca Kaßner, Stefan Seemayer, Esmeralda Vicedo, Christian Schaefer, Dominik Achten, Florian Auer, Ariane Boehm, Tatjana Braun, Maximilian Hecht, Mark Heron, Peter Hönigschmid, Thomas A Hopf, Stefanie Kaufmann, Michael Kiening, Denis Krompass, Cedric Landerer, Yannick Mahlich, Manfred Roos, Jari Björne, Tapio Salakoski, Andrew Wong, Hagit Shatkay, Fanny Gatzmann, Ingolf Sommer, Mark N Wass, Michael J E Sternberg, Nives Škunca, Fran Supek, Matko Bošnjak, Panče Panov, Sašo Džeroski, Tomislav Šmuc, Yiannis A I Kourmpetis, Aalt D J van Dijk, Cajo J F ter Braak, Yuanpeng Zhou, Qingtian Gong, Xinran Dong, Weidong Tian, Marco Falda, Paolo Fontana, Enrico Lavezzo, Barbara Di Camillo, Stefano Toppo, Liang Lan, Nemanja Djuric, Yuhong Guo, Slobodan Vucetic, Amos Bairoch, Michal Linial, Patricia C Babbitt, Steven E Brenner, Christine Orengo, Burkhard Rost, Sean D Mooney, and Iddo Friedberg. A large-scale evaluation of computational protein function prediction. Nature Methods, 10(3):221-227, January 2013. doi: 10.1038/nmeth.2340. URL https://doi.org/10.1038/nmeth.2340. 1

[7] Philip A Romero and Frances H Arnold. Exploring protein fitness landscapes by directed evolution. Nature reviews Molecular cell biology, 10(12):866-876, 2009. 1

[8] R. Fox, A. Roy, S. Govindarajan, J. Minshull, C. Gustafsson, J. T. Jones, and R. Emig. Optimizing the search algorithm for protein engineering by directed evolution. Protein Engineering Design and Selection, 16(8):589-597, August 2003. doi: 10.1093/protein/gzg077. URL https://doi.org/10.1093/protein/gzg077. 1

[9] Kevin K Yang, Zachary Wu, and Frances H Arnold. Machine-learning-guided directed evolution for protein engineering. Nature methods, 16(8):687-694, 2019. 1]

[10] Zachary Wu, SB Jennifer Kan, Russell D Lewis, Bruce J Wittmann, and Frances H Arnold. Machine learning-assisted directed protein evolution with combinatorial libraries. Proceedings of the National Academy of Sciences, 116(18):8852-8858, 2019. 3.1

[11] Bruce J. Wittmann, Yisong Yue, and Frances H. Arnold. Machine learning-assisted directed evolution navigates a combinatorial epistatic fitness landscape with minimal screening burden. bioRxiv, 2020. doi: 10.1101/2020.12.04.408955. URL https://www.biorxiv.org/ content/early/2020/12/04/2020.12.04.408955. 1

[12] Martin Steinegger and Johannes Söding. MMseqs2 enables sensitive protein sequence searching for the analysis of massive data sets. Nature biotechnology, 35(11):1026-1028, 2017. 11, 3.3

[13] Martin Steinegger and Johannes Söding. Clustering huge protein sequence sets in linear time. Nature communications, 9(1):1-8, 2018. 1]

[14] UniProt: the universal protein knowledgebase in 2021. Nucleic Acids Research, 49(D1): D480-D489, 2021. 1, 3.2 
[15] Ahmed Elnaggar, Michael Heinzinger, Christian Dallago, Ghalia Rehawi, Wang Yu, Llion Jones, Tom Gibbs, Tamas Feher, Christoph Angerer, Martin Steinegger, Debsindhu Bhowmik, and Burkhard Rost. ProtTrans: Towards cracking the language of lifes code through selfsupervised deep learning and high performance computing. IEEE Transactions on Pattern Analysis and Machine Intelligence, pages 1-1, 2021. doi: 10.1109/tpami.2021.3095381. URL https://doi.org/10.1109/tpami.2021.3095381. 1, 3

[16] Joshua Meier, Roshan Rao, Robert Verkuil, Jason Liu, Tom Sercu, and Alexander Rives. Language models enable zero-shot prediction of the effects of mutations on protein function. bioRxiv, 2021. 1, 2, 3, 5, 5

[17] Surojit Biswas, Grigory Khimulya, Ethan C Alley, Kevin M Esvelt, and George M Church. Low-N protein engineering with data-efficient deep learning. Nature Methods, 18(4):389-396, 2021. [1]

[18] Thomas A Hopf, John B Ingraham, Frank J Poelwijk, Charlotta PI Schärfe, Michael Springer, Chris Sander, and Debora S Marks. Mutation effects predicted from sequence co-variation. Nature biotechnology, 35(2):128-135, 2017. 1

[19] Adam J Riesselman, John B Ingraham, and Debora S Marks. Deep generative models of genetic variation capture the effects of mutations. Nature methods, 15(10):816-822, 2018. 2, 3]

[20] Jonathan Frazer, Pascal Notin, Mafalda Dias, Aidan Gomez, Kelly Brock, Yarin Gal, and Debora Marks. Large-scale clinical interpretation of genetic variants using evolutionary data and deep learning. bioRxiv, 2020.

[21] Jung-Eun Shin, Adam J Riesselman, Aaron W Kollasch, Conor McMahon, Elana Simon, Chris Sander, Aashish Manglik, Andrew C Kruse, and Debora S Marks. Protein design and variant prediction using autoregressive generative models. Nature communications, 12(1):1-11, 2021. 11

[22] Andriy Kryshtafovych, Torsten Schwede, Maya Topf, Krzysztof Fidelis, and John Moult. Critical assessment of methods of protein structure prediction (CASP)-Round XIII. Proteins: Structure, Function, and Bioinformatics, 87(12):1011-1020, 2019. 2

[23] Mohammed AlQuraishi. ProteinNet: a standardized data set for machine learning of protein structure. BMC bioinformatics, 20(1):1-10, 2019. 2

[24] Masthead. Proteins: Structure, Function, and Bioinformatics, 23(3):fmi-fmi, 1995. doi: https://doi.org/10.1002/prot.340230301. URL https://onlinelibrary.wiley.com/doi/ abs/10.1002/prot.340230301. 2

[25] John Jumper, Richard Evans, Alexander Pritzel, Tim Green, Michael Figurnov, Olaf Ronneberger, Kathryn Tunyasuvunakool, Russ Bates, Augustin Žídek, Anna Potapenko, et al. Highly accurate protein structure prediction with AlphaFold. Nature, pages 1-11, 2021. 2

[26] Alexander Rives, Joshua Meier, Tom Sercu, Siddharth Goyal, Zeming Lin, Jason Liu, Demi Guo, Myle Ott, C Lawrence Zitnick, Jerry Ma, et al. Biological structure and function emerge from scaling unsupervised learning to 250 million protein sequences. Proceedings of the National Academy of Sciences, 118(15), 2021. 1, 3, 3, 5, 5

[27] Roshan Rao, Nicholas Bhattacharya, Neil Thomas, Yan Duan, Xi Chen, John Canny, Pieter Abbeel, and Yun S Song. Evaluating protein transfer learning with TAPE. Advances in neural information processing systems, 32:9689, 2019. 1, 2, 3

[28] Tristan Bepler and Bonnie Berger. Learning protein sequence embeddings using information from structure. In International Conference on Learning Representations, 2018. 1

[29] Amy X Lu, Haoran Zhang, Marzyeh Ghassemi, and Alan M Moses. Self-supervised contrastive learning of protein representations by mutual information maximization. BioRxiv, 2020. 1]

[30] Amir Shanehsazzadeh, David Belanger, and David Dohan. Is transfer learning necessary for protein landscape prediction? arXiv preprint arXiv:2011.03443, 2020. 1. 2 
[31] Chloe Hsu, Hunter Nisonoff, Clara Fannjiang, and Jennifer Listgarten. Combining evolutionary and assay-labelled data for protein fitness prediction. bioRxiv, 2021. 1]

[32] Naihui Zhou, Yuxiang Jiang, Timothy R Bergquist, Alexandra J Lee, Balint Z Kacsoh, Alex W Crocker, Kimberley A Lewis, George Georghiou, Huy N Nguyen, Md Nafiz Hamid, et al. The CAFA challenge reports improved protein function prediction and new functional annotations for hundreds of genes through experimental screens. Genome biology, 20(1):1-23, 2019. 2

[33] Christophe Dessimoz, Nives Škunca, and Paul D Thomas. CAFA and the open world of protein function predictions. Trends in Genetics, 29(11):609-610, 2013. 2

[34] Tristan Bepler and Bonnie Berger. Learning the protein language: Evolution, structure, and function. Cell Systems, 12(6):654-669.e3, June 2021. doi: 10.1016/j.cels.2021.05.017. URL https://doi.org/10.1016/j.cels.2021.05.017. 2, 3

[35] Vanessa E Gray, Ronald J Hause, Jens Luebeck, Jay Shendure, and Douglas M Fowler. Quantitative missense variant effect prediction using large-scale mutagenesis data. Cell systems, 6(1): 116-124, 2018. 2, 3

[36] Roshan Rao, Joshua Meier, Tom Sercu, Sergey Ovchinnikov, and Alexander Rives. Transformer protein language models are unsupervised structure learners. In International Conference on Learning Representations, 2020. 2

[37] Michael Heinzinger, Ahmed Elnaggar, Yu Wang, Christian Dallago, Dmitrii Nechaev, Florian Matthes, and Burkhard Rost. Modeling aspects of the language of life through transferlearning protein sequences. BMC Bioinformatics, 20(1), December 2019. doi: 10.1186/ s12859-019-3220-8. URL https://doi.org/10.1186/s12859-019-3220-8. 2, 3

[38] Céline Marquet, Michael Heinzinger, Tobias Olenyi, Christian Dallago, Michael Bernhofer, Kyra Erckert, and Burkhard Rost. Embeddings from protein language models predict conservation and variant effects. 2021. [2

[39] Maria Littmann, Michael Heinzinger, Christian Dallago, Tobias Olenyi, and Burkhard Rost. Embeddings from deep learning transfer GO annotations beyond homology. Scientific reports, 11(1):1-14, 2021.

[40] Hannes Stärk, Christian Dallago, Michael Heinzinger, and Burkhard Rost. Light attention predicts protein location from the language of life. bioRxiv, 2021. 2

[41] Nicholas C Wu, Lei Dai, C Anders Olson, James O Lloyd-Smith, and Ren Sun. Adaptation in protein fitness landscapes is facilitated by indirect paths. Elife, 5:e16965, 2016. 1, 3, 3.1]

[42] W. Trent Franks, Benjamin J. Wylie, Sara A. Stellfox, and Chad M. Rienstra. Backbone conformational constraints in a microcrystalline $\mathrm{u}-15 \mathrm{n}$-labeled protein by $3 \mathrm{~d}$ dipolar-shift solidstate nmr spectroscopy. Journal of the American Chemical Society, 128(10):3154-3155, 2006. doi: 10.1021/ja058292x. URL https://doi.org/10.1021/ja058292x. PMID: 16522090. 1

[43] Drew H Bryant, Ali Bashir, Sam Sinai, Nina K Jain, Pierce J Ogden, Patrick F Riley, George M Church, Lucy J Colwell, and Eric D Kelsic. Deep diversification of an AAV capsid protein by machine learning. Nature Biotechnology, 39(6):691-696, 2021. 1, 3, 3.2

[44] Ran Zhang, Lin Cao, Mengtian Cui, Zixian Sun, Mingxu Hu, Rouxuan Zhang, William Stuart, Xiaochu Zhao, Zirui Yang, Xueming Li, Yuna Sun, Shentao Li, Wei Ding, Zhiyong Lou, and Zihe Rao. Adeno-associated virus 2 bound to its cellular receptor AAVR. Nature Microbiology, 4(4):675-682, February 2019. doi: 10.1038/s41564-018-0356-7. URL https://doi.org/ 10.1038/s41564-018-0356-7. 1

[45] Anna Jarzab, Nils Kurzawa, Thomas Hopf, Matthias Moerch, Jana Zecha, Niels Leijten, Yangyang Bian, Eva Musiol, Melanie Maschberger, Gabriele Stoehr, et al. Meltome atlasthermal proteome stability across the tree of life. Nature methods, 17(5):495-503, 2020. [1, 3 , 3.3 
[46] Peer Bork and Eugene V Koonin. Predicting functions from protein sequences—where are the bottlenecks? Nature genetics, 18(4):313-318, 1998. 3

[47] A Elisabeth Sauer-Eriksson, Gerard J Kleywegt, Mathias Uhlén, and T Alwyn Jones. Crystal structure of the $\mathrm{C} 2$ fragment of streptococcal protein $\mathrm{G}$ in complex with the Fc domain of human IgG. Structure, 3(3):265-278, 1995. 3.1

[48] U Sjöbring, L Björck, and W Kastern. Streptococcal protein G. Gene structure and protein binding properties. Journal of Biological Chemistry, 266(1):399-405, 1991. 3.1

[49] LH Vandenberghe, JM Wilson, and G Gao. Tailoring the AAV vector capsid for gene therapy. Gene therapy, 16(3):311-319, 2009. 3.2

[50] Hildegard Büning, Anke Huber, Liang Zhang, Nadja Meumann, and Ulrich Hacker. Engineering the AAV capsid to optimize vector-host-interactions. Current opinion in pharmacology, 24: 94-104, 2015. 3.2

[51] Christopher Barnes, Olivia Scheideler, and David Schaffer. Engineering the AAV capsid to evade immune responses. Current opinion in biotechnology, 60:99-103, 2019. 3.2

[52] Georgios Mikos, Weitong Chen, and Junghae Suh. Machine learning identification of capsid mutations to improve AAV production fitness. bioRxiv, 2021. 3.2

[53] Sam Sinai, Nina Jain, George M Church, and Eric D Kelsic. Generative AAV capsid diversification by latent interpolation. bioRxiv, 2021. 3.2

[54] Ryan Lauchli, Kersten S Rabe, Karolina Z Kalbarczyk, Amulya Tata, Thomas Heel, Rebekah Z Kitto, and Frances $\mathrm{H}$ Arnold. High-throughput screening for terpene-synthase-cyclization activity and directed evolution of a terpene synthase. Angewandte Chemie International Edition, 52(21):5571-5574, 2013. 3.3

[55] J. D. Bloom, S. T. Labthavikul, C. R. Otey, and F. H. Arnold. Protein stability promotes evolvability. Proceedings of the National Academy of Sciences, 103(15):5869-5874, March 2006. doi: 10.1073/pnas.0510098103. URL https://doi.org/10.1073/pnas.0510098103. 3.3

[56] Yoshiaki Nosoh and Takeshi Sekiguchi. Protein engineering for thermostability. Trends in biotechnology, 8(1):16-20, 1990. 3.3

[57] Yougen Li, D Allan Drummond, Andrew M Sawayama, Christopher D Snow, Jesse D Bloom, and Frances $\mathrm{H}$ Arnold. A diverse family of thermostable cytochrome P450s created by recombination of stabilizing fragments. Nature biotechnology, 25(9):1051-1056, 2007.

[58] Pete Heinzelman, Christopher D Snow, Indira Wu, Catherine Nguyen, Alan Villalobos, Sridhar Govindarajan, Jeremy Minshull, and Frances H Arnold. A family of thermostable fungal cellulases created by structure-guided recombination. Proceedings of the National Academy of Sciences, 106(14):5610-5615, 2009. 3.3

[59] Margaux M Pinney, Daniel A Mokhtari, Eyal Akiva, Filip Yabukarski, David M Sanchez, Ruibin Liang, Tzanko Doukov, Todd J Martinez, Patricia C Babbitt, and Daniel Herschlag. Parallel molecular mechanisms for enzyme temperature adaptation. Science, 371(6533), 2021. 3.3

[60] F. Y. Wu. The potts model. Rev. Mod. Phys., 54:235-268, Jan 1982. doi: 10.1103/RevModPhys. 54.235. URL https://link.aps.org/doi/10.1103/RevModPhys.54.235. 4

[61] Faruck Morcos, Andrea Pagnani, Bryan Lunt, Arianna Bertolino, Debora S. Marks, Chris Sander, Riccardo Zecchina, José N. Onuchic, Terence Hwa, and Martin Weigt. Direct-coupling analysis of residue coevolution captures native contacts across many protein families. Proceedings of the National Academy of Sciences, 108(49):E1293-E1301, 2011. ISSN 0027-8424. doi: 10.1073/pnas.1111471108. URL https://www . pnas .org/content/108/49/E1293. 4

[62] William P Russ, Matteo Figliuzzi, Christian Stocker, Pierre Barrat-Charlaix, Michael Socolich, Peter Kast, Donald Hilvert, Remi Monasson, Simona Cocco, Martin Weigt, et al. An evolutionbased model for designing chorismate mutase enzymes. Science, 369(6502):440-445, 2020. 田

[63] Diederik P Kingma and Jimmy Ba. Adam: A method for stochastic optimization. arXiv preprint arXiv:1412.6980, 2014. 4 